\title{
Planetary Systems Around Red Giants and Formation of Planetary Nebulae
}

\author{
G.M. Rudnitskij \\ Sternberg Astronomical Institute, 13 Universitetskij prospekt \\ Moscow 119899 Russia
}

\begin{abstract}
The effects of planetary systems around pre-AGB and AGB stars on their pulsations, optical spectra and on the shape of future planetary nebulae are discussed. The physical phenomena considered include include gas drag on the planet embedded in the ever denser circumstellar gas of a red giant and shock waves arising. intensity of the $\mathrm{H}_{2} \mathrm{O}$ The lack of variability in many red giants may be due to their low metallicity and absence of planetary systems around them.
\end{abstract}

\section{Introduction}

At present, more than 70 main-sequence stars in the solar vicinity have shown the presence of planetary systems (PS, Schneider 2002), which are absolutely dissimilar to the Solar System. Most of them are Jupiter-mass objects, revolving in orbits very close to the stars $\left(\sim 0.05-0.1 \mathrm{AU}, P_{\text {orb }} \sim\right.$ a few days - 'hot Jupiters') or in highly eccentric orbits with $e$ up to 0.7 . There is no doubt that future searches will detect lower-mass and longer-period planets, probably of the terrestrial type.

If a star possessed a PS during its main-sequence life, at the red-giant stage the closer-by planets, revolving at $R \sim 1-3 \mathrm{AU}$, will be embedded within the star's atmosphere. Below some effects of PS around red giants (planetary nebula progenitors) are discussed.

\section{Planets and Mira Variables}

It is commonly accepted that the long-period variability of red giants - Miratype and semiregulars - is caused by stellar pulsations. Their periods $P$ are mainly concentrated between 150 and 600 days. Soker (1992) stated that a planet that is still orbiting inside the red-giant atmosphere (or even deeper) could contribute to excitation of stellar pulsations (though probably nonradial, hence smaller-amplitude ones). Intrinsic pulsations of red giants may be rather weak and irregular. Perhaps, a planet with $P_{\text {orb }}=150-600^{d}$ is the organising factor of the long-period pulsations.

Usually the optical emission lines appearing in Miras' spectra are interpreted as due to pulsation-driven shocks, though recent data (Reid \& Menten 1997) probably indicate that spherical shocks cannot account for the observed emission. I propose to explain the optical emission by a local hot spot in the 
stellar atmosphere, due to a supersonic motion of a planet. A strong conical shock wave is formed. The shocked cone trailing behind the planet is a source of optical emission lines, it may be also observable as a hot spot migrating over the stellar disc (López et al. 1997).

Another case is a very eccentric orbit with $P \sim 10-15$ years. Esipov et al. (1999) have monitored the spectra of a sample of Miras since 1994. The main aim was to search for correlation with the variations of the circumstellar $\mathrm{H}_{2} \mathrm{O}$ maser emission $(\lambda=1.35 \mathrm{~cm})$ in Miras. Some stars displayed isolated bursts of the $\mathrm{H} \alpha$ emission, followed (about a year and a half later) by a flare of the $\mathrm{H}_{2} \mathrm{O}$ maser radio emission. These stars are $\mathrm{R}$ Leo, $\mathrm{R}$ Cas and $\mathrm{U}$ Aur. We have been tracing their $\mathrm{H}_{2} \mathrm{O}$ maser history since early 1980s (Berulis et al. 1983), when they were rather strong $\mathrm{H}_{2} \mathrm{O}$ emitters, but soon faded and remained silent in the $\mathrm{H}_{2} \mathrm{O}$ line until 1997-1998. Then the probably shock-stimulated $\mathrm{H}_{2} \mathrm{O}$ flares happened (see model by Rudnitskij \& Chuprikov 1990). Interpreting this in the framework of our model, this may be a periastron episode of a planet in a highly eccentric orbit with a period $P \sim 15$ years.

\section{Planets and stellar metallicities}

As stated above, the presence of relic PS may account for the Mira-type variability itself, while nonvariable red giants are those devoid of PS. This view is supported by the lack of Miras among low-metallicity stars - in the halo and in low-metallicity (probably all) globular clusters (Barthès \& Luri 2001, Mennessier et al. 2001: a low abundance of heavy elements prevents PS formation. The above PS effects result in a pronounced asymmetry of the mass loss outflow and in quaint $\mathrm{PN}$ shapes. The asymmetric structures recently observed by IR and $\mathrm{OH} / \mathrm{H}_{2} \mathrm{O}$ maser interferometry around some AGB stars are discussed in this context. In particular, the statistics of the LMC PN shapes (Stanghellini et al. 2000) shows that strongly asymmetric PN may have formed from more massive (hence younger and metal-richer) stars, thus also inferring probable importance of PS around the PN progenitors for the onset of the nonspherical mass loss.

\section{References}

Barthès, D., \& Luri, X. 2001, A\&A, 365, 519

Berulis, I. I., Lekht, E. E., Pashchenko, M. I., \&

Rudnitskij, G. M. 1983, Soviet Ast., 27, 179

Esipov, V. F., Pashchenko, M. I., Rudnitskij, G. M., \& Fomin, S. V. 1999, Astron. Lett., 25, 672

López, B. et al. 1997, ApJ, 488, 807

Mennessier, M. O., Mowlavi, N., Alvarez, R., \& Luri, X. 2001, A\&A, 374, 968

Reid, M. J., \& Menten, K. M. 1997, ApJ, 476, 327

Rudnitskij, G. M., \& Chuprikov, A. A. 1990, Soviet Ast., 34, 147

Schneider, J. 2002, http://www.obspm.fr/planets/catalog.html

Soker, N. 1992, ApJ, 386, 190

Soker, N. 1999, MNRAS, 306, 806

Stanghellini, L., Shaw, R. A., Balick, B., \& Blades, J. C. 2000, ApJ, 534, L167 